International Journal of Pure and Applied Mathematics

Volume 104 No. 4 2015, 561-575

ISSN: 1311-8080 (printed version); ISSN: 1314-3395 (on-line version)

url: http://www.ijpam.eu

doi: http://dx.doi.org/10.12732/ijpam.v104i4.8

ijpam.eu

\title{
AN ALGORITHM FOR SOLVING ASSIGNMENT PROBLEMS WITH COSTS AS GENERALIZED TRAPEZOIDAL INTUITIONISTIC FUZZY NUMBERS
}

\author{
A. Nagoor Gani ${ }^{1}$, V.N. Mohamed ${ }^{2}$ \\ ${ }^{1,2} \mathrm{PG}$ and Research Department of Mathematics \\ Jamal Mohamed College (Autonomous) \\ Tiruchirappalli, 620020, INDIA
}

\begin{abstract}
Assignment problem is a well-known topic and is used very often in solving problems of engineering and management sciences. In this problem $c_{i j}$ denotes the cost for assigning the $j$ th job to the $i$ th person. This cost is usually deterministic in nature. In this paper $c_{i j}$ has been considered to be generalized trapezoidal intuitionistic fuzzy numbers denoted by $\tilde{c}_{i j}$ which are more realistic and general in nature. Here, we present a new algorithm for solving assignment problems whose costs as generalized trapezoidal intuitionistic fuzzy numbers. We developed the algorithm using the fundamental theorem of fuzzy assignment problems.
\end{abstract}

AMS Subject Classification: 03E72, 90C08

Key Words: assignment problem, Intuitionistic fuzzy numbers, generalized trapezoidal intuitionistic fuzzy numbers, ranking of fuzzy numbers

\section{Introduction}

The Assignment Problem (AP) is one of the well studied optimization problems in engineering and management sciences and has been widely applied in both

Received: June 25, 2015

(C) 2015 Academic Publications, Ltd.

${ }^{\S}$ Correspondence author url: www.acadpubl.eu 
manufacturing and service systems. In an assignment problem, $n$ jobs are to be performed by $n$ persons depending on their efficiency to do the job. In this problem $c_{i j}$ denotes the cost of assigning the $j$ th job to the $i$ th person. We assume that one person can be assigned exactly one job; also each person can do at most one job. The problem is to find an optimal assignment so that the total cost of performing all jobs is minimum or the total profit is maximum.

To find solutions to assignment problems, various algorithms such as linear programming, Hungarian algorithm, neural network and genetic algorithm have been developed. Over the past 50 years, many variations of the classical assignment problems are proposed, e.g., bottleneck assignment problem, quadratic assignment problem etc. Lin and Wen [5] proposed an efficient algorithm based on the labeling method for solving the linear fractional programming case. Sakawa et al. [10] solved the problems on production and work force assignment in a firm using interactive fuzzy programming for two level linear and linear fractional programming models. Chen [3] projected a fuzzy assignment model that considers all persons to have same skills. Chen Liang-Hsuan and Lu Hai-Wen [4] developed a procedure for solving assignment problems with multiple inadequate inputs and outputs in crisp form for each possible assignment using linear programming model to determine the assignments with maximum efficiency. Linzhong Liu and Xin Goa [6] considered the genetic algorithm for solving the fuzzy weighted equilibrium and multi-job assignment problem. Majumdar and Bhunia [8] developed an exclusive genetic algorithm to solve a generalized assignment problem with imprecise $\operatorname{cost}(\mathrm{s}) / \operatorname{time}(\mathrm{s})$, in which the impreciseness of $\operatorname{cost}(\mathrm{s}) / \operatorname{time}(\mathrm{s})$ are represented by interval valued numbers. Xionghui Ye and Jiuping Xu [17] developed a priority based genetic algorithm to a fuzzy vehicle routing assignment model with connection network. Sathi Mukherjee and Kajla Basu [13] developed a method for solving intuitionistic fuzzy assignment problems by using similarity measures and score function. Pandian and Kavitha [12], Shiny Jose and Sunny Kuriakose [14], Thorani and Ravi Shankar [16], and Nirmala and Anju [11] developed various algorithms for solving assignment problems in the fuzzy context. Here we are considering assignment problems having generalized trapezoidal intuitionistic fuzzy numbers as costs. We apply a ranking method [9] defined on generalized intuitionistic trapezoidal fuzzy numbers to rank the fuzzy costs present in the assignment problem.

This paper is organized as follows. In Section 2, we present the basic concepts of generalized trapezoidal intuitionistic fuzzy numbers and described the arithmetic operations of such numbers. In Section 3, a ranking method is given to rank the generalized trapezoidal intuitionistic fuzzy numbers. In Section 4, 
intuitionistic fuzzy assignment problem, mathematical formulation of intuitionistic fuzzy assignment problem and fundamental theorem of intuitionistic fuzzy assignment problem are reviewed. Section 5 presents an algorithm for solving an assignment problem with costs as generalized trapezoidal intuitionistic fuzzy numbers. In Section 6, numerical example is presented to show the application of the proposed algorithm. Finally, the conclusion is given in Section 7 .

\section{Preliminaries on Intuitionistic Fuzzy Sets and Intuitionistic Fuzzy Numbers}

In this section we will review the basic concepts of intuitionistic fuzzy sets and intuitionistic fuzzy numbers

\subsection{Intuitionistic Fuzzy Sets}

Definition $2.1([1,2])$. Let $X$ be the universal set. An intuitionistic fuzzy set (IFS) $A$ in $X$ is given by

$$
A=\left\{\left(x, \mu_{A}(x), \nu_{A}(x)\right): x \in X\right\}
$$

where the functions $\mu_{A}(x), \nu_{A}(x)$ define respectively, the degree of membership and degree of non-membership of the element $x \in X$ to the set $A$, which is a subset of $X$, and for every $x \in X, 0 \leq \mu_{A}(x)+\nu_{A}(x) \leq 1$.

Obviously, every fuzzy set has the form

$$
A=\left\{\left(x, \mu_{A}(x), \mu_{A^{c}}(x)\right): x \in X\right\} \text { in } X .
$$

For each intuitionistic fuzzy set $A=\left\{\left(x, \mu_{A}(x), \nu_{A}(x)\right): x \in X\right.$,

$$
\pi_{A}(x)=1-\mu_{A}(x)-\nu_{A}(x)
$$

is called the hesitancy degree of $x$ to lie in $A$. If $A$ is a fuzzy set, then $\pi_{A}(x)=0$ for all $x \in X$.

\subsection{Intuitionistic Fuzzy Numbers}

Here we will introduce the intuitionistic fuzzy number (IFN) [7] and generalized trapezoidal intuitionistic fuzzy number (GTIFN) [15] and some properties of them. 
Definition 2.2. An IFS $A=\left\{\left(x, \mu_{A}(x), \nu_{A}(x)\right): x \in X\right\}$ of the real line $\mathbb{R}$ is called an intuitionistic fuzzy number (IFN) if

(a) $A$ is convex for the membership function $\mu_{A}(x)$, i.e., if $\mu_{A}\left(\lambda x_{1}+(1-\lambda) x_{2}\right) \geq \mu_{A}\left(x_{1}\right) \wedge \mu_{A}\left(x_{2}\right)$ for all $x_{1}, x_{2} \in \mathbb{R}$, $\lambda \in[0,1]$.

(b) $A$ is concave for the non-membership function $\nu_{A}(x)$, i.e., if $\nu_{A}\left(\lambda x_{1}+(1-\lambda) x_{2}\right) \leq \nu_{A}\left(x_{1}\right) \vee \nu_{A}\left(x_{2}\right)$ for all $x_{1}, x_{2} \in \mathbb{R}$, $\lambda \in[0,1]$.

(c) $A$ is normal, that is, there is some $x_{0} \in \mathbb{R}$ such that $\mu_{A}\left(x_{0}\right)=1$ and $\nu_{A}\left(x_{0}\right)=0$.

Definition 2.3. An intuitionistic fuzzy number $A$ is said to be a generalized trapezoidal intuitionistic fuzzy number (GTIFN) with parameters

$$
b_{1} \leq a_{1} \leq b_{2} \leq a_{2} \leq a_{3} \leq b_{3} \leq a_{4} \leq b_{4},
$$

and denoted by

$$
A=\left(b_{1}, a_{1}, b_{2}, a_{2}, a_{3}, b_{3}, a_{4}, b_{4} ; \omega_{A}, u_{A}\right)
$$

or

$$
A=\left(\left(a_{1}, a_{2}, a_{3}, a_{4}\right),\left(b_{1}, b_{2}, b_{3}, b_{4}\right) ; \omega_{A}, u_{A}\right)
$$

if its membership and non-membership functions are as follows:

$$
\mu_{A}(x)= \begin{cases}0, & \text { if } x<a_{1} \\ \omega_{A}\left(\frac{x-a_{1}}{a_{2}-a_{1}}\right), & \text { if } a_{1} \leq x \leq a_{2} \\ \omega_{A}, & \text { if } a_{2} \leq x \leq a_{3} \\ \omega_{A}\left(\frac{a_{4}-x}{a_{4}-a_{3}}\right), & \text { if } a_{3} \leq x \leq a_{4} \\ 0, & \text { if } x>a_{4} .\end{cases}
$$

and

$$
\nu_{A}(x)= \begin{cases}1, & \text { if } x<b_{1} \\ \frac{\left(b_{2}-x\right)+u_{\mathrm{A}}\left(x-b_{1}\right)}{b_{2}-b_{1}}, & \text { if } b_{1} \leq x \leq b_{2} \\ u_{A}, & \text { if } b_{2} \leq x \leq b_{3} \\ \frac{\left(x-b_{3}\right)+u_{\mathrm{A}}\left(b_{4}-x\right)}{b_{4}-b_{3}}, & \text { if } b_{3} \leq x \leq b_{4} \\ 1, & \text { if } x>b_{4} .\end{cases}
$$

where $0<\omega_{A} \leq 1,0 \leq u_{A} \leq 1$ and $0<\omega_{A}+u_{A} \leq 1$. 


\subsection{Arithmetic Operations on Generalized Trapezoidal Intuitionistic Fuzzy Numbers}

$$
\begin{aligned}
\text { Let } A & =\left(\left(a_{1}, a_{2}, a_{3}, a_{4}\right),\left(b_{1}, b_{2}, b_{3}, b_{4}\right) ; \omega_{A}, u_{A}\right) \\
\text { and } B & =\left(\left(c_{1}, c_{2}, c_{3}, c_{4}\right),\left(d_{1}, d_{2}, d_{3}, d_{4}\right) ; \omega_{B}, u_{B}\right)
\end{aligned}
$$

be two GTIFNs and $\lambda$ be a real number. Then

(i) $A+B=\left(\left(a_{1}+c_{1}, a_{2}+c_{2}, a_{3}+c_{3}, a_{4}+c_{4}\right)\right.$,

$$
\left.\left(b_{1}+d_{1}, b_{2}+d_{2}, b_{3}+d_{3}, b_{4}+d_{4}\right) ; \omega, u\right)
$$

where $\omega=\min \left\{\omega_{A}, \omega_{B}\right\}$ and $u=\max \left\{u_{A}, u_{B}\right\}$.

(ii) $A-B=\left(\left(a_{1}-c_{4}, a_{2}-c_{3}, a_{3}-c_{2}, a_{4}-c_{1}\right)\right.$,

$$
\left.\left(b_{1}-d_{4}, b_{2}-d_{3}, b_{3}-d_{2}, b_{4}-d_{1}\right) ; \omega, u\right)
$$

where $\omega=\min \left\{\omega_{A}, \omega_{B}\right\}$ and $u=\max \left\{u_{A}, u_{B}\right\}$

(iii) $\quad \lambda A=\left(\left(\lambda a_{1}, \lambda a_{2}, \lambda a_{3} \lambda a_{4}\right),\left(\lambda b_{1}, \lambda b_{2}, \lambda b_{3} \lambda b_{4}\right) ; \omega_{A}, u_{A}\right)$

if $\lambda>0$

$=\left(\left(\lambda a_{4}, \lambda a_{3}, \lambda a_{2}, \lambda a_{1}\right),\left(\lambda b_{4}, \lambda b_{3}, \lambda b_{2} b a_{1}\right) ; \omega_{A}, u_{A}\right)$

if $\lambda<0$.

(iv) $A \otimes B=\left(\left(a_{1} c_{1}, a_{2} c_{2}, a_{3} c_{3}, a_{4} c_{4}\right),\left(b_{1} d_{1}, b_{2} d_{2} b_{3} d_{3} b_{4} d_{4}\right) ; \omega, u\right)$

if $A>0, B>0$

$=\left(\left(a_{1} c_{4}, a_{2} c_{3}, a_{3} c_{2}, a_{4} c_{1}\right),\left(b_{1} d_{4}, b_{2} d_{3}, b_{3} d_{2}, b_{4} d_{1}\right) ; \omega, u\right)$

if $A<0, B>0$

$=\left(\left(a_{4} c_{4}, a_{3} c_{3}, a_{2} c_{2}, a_{1} c_{1}\right),\left(b_{4} d_{4}, b_{3} d_{3}, b_{2} d_{2}, b_{1} d_{1}\right) ; \omega, u\right)$

if $A<0, B<0$

where $\omega=\min \left\{\omega_{A}, \omega_{B}\right\}$ and $u=\max \left\{u_{A}, u_{B}\right\}$

(v) $A \div B=\left(\left(\frac{a_{1}}{c_{4}}, \frac{a_{2}}{c_{3}}, \frac{a_{3}}{c_{2}}, \frac{a_{4}}{c_{1}}\right),\left(\frac{b_{1}}{d_{4}}, \frac{b_{2}}{d_{3}}, \frac{b_{3}}{d_{2}}, \frac{b_{4}}{d_{1}}\right), \omega, u\right)$

where $u=\min \left\{\omega_{A}, \omega_{B}\right\}$ and $u=\max \left\{u_{A}, u_{B}\right\}$, if $d_{1}>0$. 


\section{Ranking of Generalized Trapezoidal Intuitionistic Fuzzy Numbers}

The ranking order relation between two GTIFNs is a difficult problem. However, GTIFNs must be ranked before the action is taken by the decision maker. We use the following method for ranking generalized trapezoidal intuitionistic fuzzy numbers.

$$
\begin{aligned}
\text { If } A & =\left(\left(a_{1}, a_{2}, a_{3}, a_{4}\right),\left(b_{1}, b_{2}, b_{3}, b_{4}\right) ; \omega_{A}, u_{A}\right), \text { then } \\
\mathscr{R}(A) & =\frac{\omega_{A} S\left(\mu_{A}\right)+u_{A} S\left(\nu_{A}\right)}{\omega_{A}+u_{A}},
\end{aligned}
$$

where

$$
S\left(\mu_{A}\right)=\left(\frac{2 a_{1}+7 a_{2}+7 a_{3}+2 a_{4}}{18}\right)\left(\frac{7 \omega_{A}}{18}\right)
$$

and

$$
S\left(\nu_{A}\right)=\left(\frac{2 b_{1}+7 b_{2}+7 b_{3}+2 b_{4}}{18}\right)\left(\frac{11+7 u_{A}}{18}\right) .
$$

\section{Intuitionistic Fuzzy Assignment Problems}

Suppose there are $n$ jobs to be performed and $n$ persons are available for doing the jobs. Assume that each person can do each job at a time, depending on their efficiency to do the job. Let $\tilde{c}_{i j}$ be the intuitionistic fuzzy cost if the $i$ th person is assigned the $j$ th job. The objective is to minimize the total intuitionistic fuzzy cost of assigning all the jobs to the available persons (one job to one person).

The intuitionistic fuzzy assignment problem can be stated in the form of an $n \times n$ cost matrix $\left[\tilde{c}_{i j}\right]$ of intuitionistic fuzzy numbers as given in the following table:

\begin{tabular}{|c|c|c|c|c|c|}
\hline Persons & \multicolumn{5}{|c|}{ Jobs } \\
\hline & 1 & 2 & 3 & $\ldots$ & $n$ \\
\hline 1 & $\tilde{c}_{11}$ & $\tilde{c}_{12}$ & $\tilde{c}_{13}$ & $\ldots$ & $\tilde{c}_{1 n}$ \\
\hline 2 & $\tilde{c}_{21}$ & $\tilde{c}_{22}$ & $\tilde{c}_{23}$ & $\ldots$ & $\tilde{c}_{2 n}$ \\
\hline$\vdots$ & $\vdots$ & $\vdots$ & $\vdots$ & $\vdots$ & $\vdots$ \\
\hline$n$ & $\tilde{c}_{n 1}$ & $\tilde{c}_{n 2}$ & $\tilde{c}_{n 3}$ & $\ldots$ & $\tilde{c}_{n n}$ \\
\hline
\end{tabular}


Mathematically an intuitionistic assignment problem can be stated as

$$
\begin{array}{lr}
\text { Minimize } & \tilde{z}=\sum_{i=1}^{n} \sum_{j=1}^{n} \tilde{c}_{i j} x_{i j} \\
\text { Subject to } \quad \sum_{j=1}^{n} x_{i j}=1, i=1,2, \ldots n, \\
\sum_{i=1}^{n} x_{i j}=1, j=1,2, \ldots n
\end{array}
$$

where $x_{i j}= \begin{cases}1, & \text { if the } i \text { th person is assigned the } j \text { th job } \\ 0, & \text { otherwise }\end{cases}$

is the decision variable denoting the assignment of the person $i$ to job $j . \tilde{c}_{i j}$ is the cost of assigning the $j$ th job to the $i$ th person. The special algorithm to solve the assignment problem is based on the following theorem.

Theorem 4.1. Let $\tilde{c}_{i j}^{\prime}=\tilde{c}_{i j}-\tilde{u}_{i}-\tilde{v}_{j}$ for all $i$ and $j$, where $\tilde{u}_{i}$ and $\tilde{v}_{j}$ are intuitionistic fuzzy constants. Let $\tilde{z}^{\prime}=\sum_{i=1}^{n} \sum_{j=1}^{n} \tilde{c}_{i j}^{\prime} x_{i j}$ be minimum for $\left[x_{i j}\right]=\left[x_{i j}^{*}\right]$. Then $\tilde{z}=\sum_{i=1}^{n} \sum_{j=1}^{n} \tilde{c}_{i j} x_{i j}$ is also minimum for $\left[x_{i j}\right]=\left[x_{i j}^{*}\right]$.

Proof. We have

$$
\begin{aligned}
\tilde{z}^{\prime} & =\sum_{i=1}^{n} \sum_{j=1}^{n} \tilde{c}_{i j}^{\prime} x_{i j} \\
& =\sum_{i=1}^{n} \sum_{j=1}^{n}\left(\tilde{c}_{i j}-\tilde{u}_{i}-\tilde{v}_{j}\right) x_{i j} \\
& =\sum_{i=1}^{n} \sum_{j=1}^{n} \tilde{c}_{i j} x_{i j}-\sum_{i=1}^{n} \sum_{j=1}^{n} \tilde{u}_{i} x_{i j}-\sum_{i=1}^{n} \sum_{j=1}^{n} \tilde{v}_{j} x_{i j} \\
& =\tilde{z}-\sum_{i=1}^{n} \tilde{u}_{i}-\sum_{j=1}^{n} \tilde{v}_{j}
\end{aligned}
$$

Since $\sum_{i=1}^{n} \tilde{u}_{i}$ and $\sum_{j=1}^{n} \tilde{v}_{j}$ are independent of $x_{i j}$, if $\left[x_{i j}^{*}\right]$ make $\tilde{z}^{\prime}$ minimum, they would also make $\tilde{z}$ minimum.

This theorem enables us to add or subtract an intuitionistic fuzzy constant from any row or column of $\left[\tilde{c}_{i j}\right]$ without affecting the optimal solution. Thus in an intuitionistic fuzzy assignment problem with cost matrix $\left[\tilde{c}_{i j}\right]$, if all $\mathscr{R}\left(\tilde{c}_{i j}\right) \geq$ 
0 , then a feasible solution $\left[x_{i j}^{*}\right]$ which satisfies $\sum_{i=1}^{n} \sum_{j=1}^{n} \mathscr{R}\left(\tilde{c}_{i j}\right) x_{i j}^{*}=0$ is also an optimal solution for the problem.

\section{An Algorithm for Solving Assignment Problems with Costs as Generalized Trapezoidal Intuitionistic Fuzzy Numbers}

Step 1: First test whether the given fuzzy cost matrix is a balanced one or not. If it is a balanced one (i.e., the number of persons are equal to the number of works), then go to step 2. If it is not balanced (i.e., the number of persons are not equal to the number of works), then introduce dummy rows (or columns) with zero intuitionistic fuzzy costs so as to form a balanced one. Then go to step 2.

Step 2: Find the rank of each cell $\tilde{c}_{i j}$ of the chosen fuzzy cost matrix by using the given ranking procedure and determine the element with least rank from each row. Subtract this element of each row in the cost matrix from every element in that row.

Step 3: Find the rank of each cell $\tilde{c}_{i j}$ of the reduced fuzzy cost matrix obtained in step 2 and determine the element with least rank from each column. Subtract this element of each column in the cost matrix from every element in that column.

Step 4: Find the rank of each cell $\tilde{c}_{i j}$ of the first modified fuzzy cost matrix obtained in step 3 . Thus, the first modified matrix of ranks $\left[\mathscr{R}\left(\tilde{c}_{i j}\right)\right]$ is obtained, and some of its entries are zeros.

Step 5: Draw the least number of lines through columns and rows which will cover all zeros of the matrix $\left[\mathscr{R}\left(\tilde{c}_{i j}\right)\right]$. Let this number be $p$. Obviously $p$ cannot be greater than $n$, the order of the matrix $\left[\mathscr{R}\left(\tilde{c}_{i j}\right)\right]$. If $p=n$, the desired solution exists; if $p<n$, it does not exist and then goes to step 6 .

Step 6: Determine the minimum element in the matrix $\left[\mathscr{R}\left(\tilde{c}_{i j}\right)\right]$ which is not covered by the $p$ lines and its corresponding fuzzy element. Subtract this fuzzy element from all fuzzy elements corresponding to the elements which are uncovered by the $p$ lines and add the same fuzzy element to the fuzzy elements corresponding to the elements which are placed at the intersection of the horizontal and vertical lines. Thus, the second modified fuzzy cost matrix is obtained. 
Step 7: Find the rank of each cell $\tilde{c}_{i j}$ of the second modified fuzzy cost matrix obtained in step 6 . Thus, the second modified matrix of ranks $\left[\mathscr{R}\left(\tilde{c}_{i j}\right)\right]$ is obtained. Repeat steps 5 and 6 until we get $p=n$.

Step 8: Examine the rows of $\left[\mathscr{R}\left(\tilde{c}_{i j}\right)\right]$ successively until a row with exactly one zero is found, make an assignment there. Then cross over all zeroes lying in the corresponding column, showing that they cannot be considered for future assignment. Continue in this manner until all the rows have been examined. Repeat the same procedure for columns also. If there is more than one zero in any row column, then mark one of the zeros arbitrarily and cross all zeros in the respective row or column and repeat the above process. Thus we get one marked zero in each row and each column of $\left[\mathscr{R}\left(\tilde{c}_{i j}\right)\right]$.

Step 9: Add the intuitionistic fuzzy numbers corresponding to the cells having a marked zero to get the total optimal fuzzy cost.

\section{Remarks}

(i) If the problem is of maximization type then find the rank of each element of the chosen fuzzy cost matrix $\left[\tilde{c}_{i j}\right]$ by using the given ranking procedure and determine the element with the highest rank. Subtract each element of the cost matrix from this element. Then the problem with the modified matrix is a minimization problem.

(ii) Sometimes technical, legal or other restrictions do not permit the assignment of a particular facility to a particular job. In such cases also we use algorithm by assigning a very high intuitionistic fuzzy cost to the cells which do not permit the assignment so that the activity will be automatically excluded from the optimal solution.

\section{Numerical Example}

To illustrate the proposed algorithm, let us consider an intuitionistic fuzzy assignment problem with rows representing 4 persons A,B,C,D and columns representing the 4 jobs namely, Job1, Job2, Job3 and Job4. The cost matrix $\left[\tilde{c}_{i j}\right]$ is given whose elements are generalized trapezoidal intuitionistic fuzzy numbers. The problem is to find the optimal assignment so that the total cost of job assignment becomes minimum. 


\begin{tabular}{|c|l|l|l|l|}
\hline \multirow{2}{*}{ Persons } & \multicolumn{5}{|c|}{ Jobs } \\
\cline { 2 - 5 } & \multicolumn{1}{|c|}{1} & \multicolumn{1}{|c|}{2} & \multicolumn{1}{c|}{3} \\
\hline A & $((3,5,6,8)$, & $((5,8,11,13)$, & $((8,10,11,15)$, & $((5,8,10,12)$, \\
& $(2,4,7,10) ;$ & $(4,6,12,14) ;$ & $(7,9,13,17) ;$ & $(4,7,11,13) ;$ \\
& $0.6,0.1)$ & $0.7,0.2)$ & $0.5,0.3)$ & $0.5,0.3)$ \\
\hline B & $((7,9,10,12)$, & $((3,5,6,8)$, & $((6,8,10,12)$, & $((5,8,10,12)$, \\
& $(6,8,11,13) ;$ & $(1,4,7,10) ;$ & $(5,7,11,13) ;$ & $(4,6,11,13) ;$ \\
& $0.7,0.1)$ & $0.4,0.3)$ & $0.7,0.1)$ & $0.8,0.1)$ \\
\hline C & $((2,4,5,7)$, & $((5,7,10,12)$, & $((8,11,13,15)$, & $((4,6,7,10)$, \\
& $(1,3,6,8) ;$ & $(4,6,11,14) ;$ & $(7,9,14,16) ;$ & $(2,5,8,11) ;$ \\
& $0.6,0.1)$ & $0.7,0.1)$ & $0.6,0.2)$ & $0.8,0.1)$ \\
\hline D & $((6,8,10,12)$, & $((2,5,6,8)$, & $((5,7,10,14)$, & $((2,4,5,7)$, \\
& $(5,7,11,13) ;$ & $(1,3,7,9) ;$ & $(4,6,12,15) ;$ & $(1,3,6,8) ;$ \\
& $0.8,0.1)$ & $0.7,0.1)$ & $0.6,0.2)$ & $0.7,0.1)$ \\
\hline
\end{tabular}

Solution: Here the problem is a balanced one. Hence we first form the matrix $\left[\mathscr{R}\left(\tilde{c}_{i j}\right)\right]$ by using the given ranking method. It is given by

\begin{tabular}{|l|l|l|l|l|}
\hline \multirow{2}{*}{ Persons } & \multicolumn{4}{|c|}{ Jobs } \\
\cline { 2 - 5 } & \multicolumn{1}{|c|}{1} & \multicolumn{1}{|c|}{2} & 3 & 4 \\
\hline $\mathrm{A}$ & $\mathbf{1 . 6 2 1}$ & 3.366 & 4.366 & 3.506 \\
\hline $\mathrm{B}$ & 3.035 & $\mathbf{2 . 2 0 4}$ & 2.875 & 3.072 \\
\hline $\mathrm{C}$ & $\mathbf{1 . 3 1 8}$ & 2.724 & 4.061 & 2.298 \\
\hline $\mathrm{D}$ & 3.139 & 1.690 & 3.096 & $\mathbf{1 . 4 3 8}$ \\
\hline
\end{tabular}

Subtract the fuzzy element with minimum rank from each row from every element in that row to get the reduced fuzzy cost matrix. It is given by

\begin{tabular}{|c|l|l|l|l|}
\hline \multirow{2}{*}{ Persons } & \multicolumn{3}{|c|}{ Jobs } \\
\cline { 2 - 5 } & \multicolumn{1}{|c|}{1} & \multicolumn{1}{|c|}{3} & \multicolumn{1}{c|}{4} \\
\hline A & $((-5,-1,1,5)$, & $((-3,2,6,10)$, & $((0,4,6,12)$, & $((-3,2,5,9)$, \\
& $(-8,-3,3,8) ;$ & $(-6,-1,8,12) ;$ & $(-3,2,9,15) ;$ & $(-6,0,7,11) ;$ \\
& $0.6,0.1)$ & $0.6,0.2)$ & $0.5,0.3)$ & $0.5,0.3)$ \\
\hline B & $((-1,3,5,9)$, & $((-5,-1,1,5)$, & $((-2,2,5,9)$, & $((-3,2,5,9)$, \\
& $(-4,1,7,12) ;$ & $(-9,-3,3,9) ;$ & $(-5,0,7,12) ;$ & $(-6,-1,7,12) ;$ \\
& $0.4,0.3)$ & $0.4,0.3)$ & $0.4,0.3)$ & $0.4,0.3)$ \\
\hline C & $((-5,-1,1,5)$, & $((-2,2,6,10)$, & $((1,6,9,13)$, & $((-3,1,3,8)$, \\
& $(-7,-3,3,7) ;$ & $(-4,0,8,13) ;$ & $(-1,3,11,15) ;$ & $(-6,-1,5,10) ;$ \\
& $0.6,0.1)$ & $0.6,0.1)$ & $0.6,0.2)$ & $0.6,0.1)$ \\
\hline D & $((-1,3,6,10)$, & $((-5,0,2,6)$, & $((-2,2,6,12)$, & $((-5,-1,1,5)$, \\
& $(-3,1,8,12) ;$ & $(-7,-3,4,8) ;$ & $(-4,0,9,14) ;$ & $(-7,-3,3,7) ;$ \\
& $0.7,0.1)$ & $0.7,0.1)$ & $0.6,0.2)$ & $0.7,0.1)$ \\
\hline
\end{tabular}

The corresponding matrix of ranks is 


\begin{tabular}{|l|l|l|l|l|}
\hline \multirow{2}{*}{ Persons } & \multicolumn{5}{|c|}{ Jobs } \\
\cline { 2 - 5 } & \multicolumn{1}{|c|}{1} & \multicolumn{1}{|c|}{2} & \multicolumn{1}{c|}{3} & 4 \\
\hline $\mathrm{A}$ & $\mathbf{0}$ & 1.264 & 2.166 & 1.306 \\
\hline $\mathrm{B}$ & 1.603 & $\mathbf{0}$ & $\mathbf{1 . 4 0 3}$ & 1.237 \\
\hline $\mathrm{C}$ & 0 & 1.182 & 2.499 & 0.608 \\
\hline $\mathrm{D}$ & 1.438 & 0.252 & 1.533 & $\mathbf{0}$ \\
\hline
\end{tabular}

There are two minimum elements in column 1. Take any one arbitrarily. Subtract the fuzzy element with minimum rank from each column from every element in that column to get the first modified fuzzy cost matrix. It is given by

\begin{tabular}{|c|l|l|l|l|}
\hline \multirow{2}{*}{ Persons } & \multicolumn{5}{|c|}{ Jobs } \\
\cline { 2 - 5 } & \multicolumn{1}{|c|}{1} & \multicolumn{1}{|c|}{3} & \multicolumn{1}{c|}{4} \\
\hline $\mathrm{A}$ & $((-10,-2,2,10)$, & $((-8,1,7,15)$, & $((-9,-1,4,14)$, & $((-8,1,6,14)$, \\
& $(-16,-6,6,16) ;$ & $(-15,-4,11,21) ;$ & $(-15,-5,9,20) ;$ & $(-13,-3,10,18) ;$ \\
& $0.6,0.1)$ & $0.4,0.3)$ & $0.4,0.3)$ & $0.5,0.3)$ \\
\hline B & $((-6,2,6,14)$, & $((-10,-2,2,10)$, & $((-11,-3,3,11)$, & $((-8,1,6,14)$, \\
& $(-12,-2,10,20) ;$ & $(-18,-6,6,18) ;$ & $(-17,-7,7,17) ;$ & $(-13,-4,10,19) ;$ \\
& $0.4,0.3)$ & $0.4,0.3)$ & $0.4,0.3)$ & $0.4,0.3)$ \\
\hline C & $((-10,-2,2,10)$, & $((-7,1,7,15)$, & $((-8,1,7,15)$, & $((-8,0,4,13)$, \\
& $(-15,-6,6,15) ;$ & $(-13,-3,11,22) ;$ & $(-13,-4,11,20) ;$ & $(-13,-4,8,17) ;$ \\
& $0.6,0.1)$ & $0.4,0.3)$ & $0.4,0.3)$ & $0.6,0.1)$ \\
\hline D & $((-6,2,7,15)$, & $((-10,-1,3,11)$, & $((-11,-3,4,14)$, & $((-10,-2,2,10)$, \\
& $(-11,-2,11,20) ;$ & $(-16,-6,7,17) ;$ & $(-16,-7,9,19) ;$ & $(-14,-6,6,14) ;$ \\
& $0.6,0.1)$ & $0.4,0.3)$ & $0.4,0.3)$ & $0.7,0.1)$ \\
\hline
\end{tabular}

The corresponding first modified crisp matrix of ranks is

\begin{tabular}{|l|l|l|l|l|}
\hline \multirow{2}{*}{ Persons } & \multicolumn{5}{|c|}{ Jobs } \\
\cline { 2 - 5 } & \multicolumn{1}{|c|}{2} & \multicolumn{1}{|c|}{3} & \multicolumn{1}{|c|}{4} \\
\hline $\mathrm{A}$ & 0 & 1.403 & 0.812 & 1.306 \\
\hline $\mathrm{B}$ & 1.603 & 0 & 0 & 1.237 \\
\hline $\mathrm{C}$ & 0 & 1.638 & 1.437 & $\mathbf{0 . 6 0 8}$ \\
\hline $\mathrm{D}$ & 1.318 & 0.235 & 0.411 & 0 \\
\hline
\end{tabular}

Here the least number of lines through columns and rows which will cover all zeros of the matrix is $3<4$. The minimum element in the matrix $\left[\mathscr{R}\left(\tilde{c}_{i j}\right)\right]$ which is not covered by the 3 lines is $\mathbf{0 . 6 0 8}$ and its corresponding fuzzy element is $((-8,0,4,13),(-13,-4,8,17) ; 0.6,0.1)$ Subtract this fuzzy element from all fuzzy elements corresponding to the elements which are uncovered by the 3 lines and add the same fuzzy element to the fuzzy elements corresponding to the elements which are placed at the intersection of the horizontal and vertical lines. Thus, the second modified fuzzy cost matrix is as follows: 


\begin{tabular}{|c|l|l|l|l|}
\hline \multirow{2}{*}{ Persons } & \multicolumn{5}{|c|}{ Jobs } \\
\cline { 2 - 5 } & \multicolumn{1}{|c|}{1} & \multicolumn{1}{|c|}{2} & \multicolumn{1}{c|}{3} \\
\hline A & $((-10,-2,2,10)$, & $((-21,-3,7,23)$, & $((-22,-5,4,22)$, & $((-21,-3,6,22)$, \\
& $(-16,-6,6,16) ;$ & $(-32,-12,15,34) ;$ & $(-32,-13,13,33) ;$ & $(-30,-11,14,31) ;$ \\
& $0.6,0.1)$ & $0.4,0.3)$ & $0.4,0.3)$ & $0.5,0.3)$ \\
\hline B & $((-14,2,10,27)$, & $((-10,-2,2,10)$, & $((-11,-3,3,11)$, & $((-8,1,6,14)$, \\
& $(-25,-6,18,37) ;$ & $(-18,-6,6,18) ;$ & $(-17,-7,7,17) ;$ & $(-13,-4,10,19) ;$ \\
& $0.4,0.3)$ & $0.4,0.3)$ & $0.4,0.3)$ & $0.4,0.3)$ \\
\hline C & $((-10,-2,2,10)$, & $((-20,-3,7,23)$, & $((-21,-3,7,23)$, & $((-21,-4,4,21)$, \\
& $(-15,-6,6,15) ;$ & $(-30,-11,15,35) ;$ & $(-30,-12,15,33) ;$ & $(-30,-12,12,30) ;$ \\
& $0.6,0.1)$ & $0.4,0.3)$ & $0.4,0.3)$ & $0.6,0.1)$ \\
\hline D & $((-14,2,11,28)$, & $((-10,-1,3,11)$, & $((-11,-3,4,14)$, & $((-10,-2,2,10)$, \\
& $(-24,-6,19,37) ;$ & $(-16,-6,7,17) ;$ & $(-16,-7,9,19) ;$ & $(-14,-6,6,14) ;$ \\
& $0.6,0.1)$ & $0.4,0.3)$ & $0.4,0.3)$ & $0.7,0.1)$ \\
\hline
\end{tabular}

The corresponding matrix of ranks is

\begin{tabular}{|l|l|l|l|l|}
\hline \multirow{2}{*}{ Persons } & \multicolumn{5}{|c|}{ Jobs } \\
\cline { 2 - 5 } & \multicolumn{1}{|c|}{1} & \multicolumn{1}{|c|}{2} & \multicolumn{1}{c|}{3} & 4 \\
\hline $\mathrm{A}$ & 0 & 0.591 & $\mathbf{0 . 0 0 1}$ & 0.504 \\
\hline $\mathrm{B}$ & 2.45 & 0 & 0 & 1.237 \\
\hline $\mathrm{C}$ & 0 & 0.826 & 0.625 & 0 \\
\hline $\mathrm{D}$ & 1.926 & 0.235 & 0.411 & 0 \\
\hline
\end{tabular}

Here the least number of lines through columns and rows which will cover all zeros of the matrix is $3<4$. The minimum element in the matrix $\left[\mathscr{R}\left(\tilde{c}_{i j}\right)\right]$ which is not covered by the 3 lines is $\mathbf{0 . 0 0 1}$ and its corresponding fuzzy element is (($22,-5,4,22),(-32,-13,13,33) ; 0.4,0.3)$ Subtract this fuzzy element from all fuzzy elements corresponding to the elements which are uncovered by the 3 lines and add the same fuzzy element to the fuzzy elements corresponding to the elements which are placed at the intersection of the horizontal and vertical lines. Thus, the third modified fuzzy cost matrix is as follows: 


\begin{tabular}{|c|l|l|l|l|}
\hline \multirow{2}{*}{ Persons } & \multicolumn{5}{|c|}{ Jobs } \\
\cline { 2 - 5 } & \multicolumn{1}{|c|}{1} & \multicolumn{1}{|c|}{2} & \multicolumn{1}{c|}{3} \\
\hline A & $((-10,-2,2,10)$, & $((-43,-7,12,45)$, & $((-44,-9,9,44)$, & $((-21,-3,6,22)$, \\
& $(-16,-6,6,16) ;$ & $(-65,-25,28,66) ;$ & $(-65,-26,26,65) ;$ & $(-30,-11,14,31) ;$ \\
& $0.6,0.1)$ & $0.4,0.3)$ & $0.4,0.3)$ & $0.5,0.3)$ \\
\hline B & $((-36,-3,14,49)$, & $((-10,-2,2,10)$, & $((-11,-3,3,11)$, & $((-30,-4,10,36)$, \\
& $(-57,-19,31.70) ;$ & $(-18,-6,6,18) ;$ & $(-17,-7,7,17) ;$ & $(-45,-17,23,52) ;$ \\
& $0.4,0.3)$ & $0.4,0.3)$ & $0.4,0.3)$ & $0.4,0.3)$ \\
\hline C & $((-10,-2,2,10)$, & $((-42,-7,12,45)$, & $((-43,-7,12,45)$, & $((-21,-4,4,21)$, \\
& $(-15,-6,6,15) ;$ & $(-63,-24,28,67) ;$ & $(-63,-25,28,65) ;$ & $(-30,-12,12,30) ;$ \\
& $0.6,0.1)$ & $0.4,0.3)$ & $0.4,0.3)$ & $0.6,0.1)$ \\
\hline D & $((-14,2,11,28)$, & $((-32,-5,8,33)$, & $((-33,-7,9,36)$, & $((-10,-2,2,10)$, \\
& $(-24,-6,19,37) ;$ & $(-49,-19,20,49) ;$ & $(-49,-20,22,51) ;$ & $(-14,-6,6,14) ;$ \\
& $0.6,0.1)$ & $0.4,0.3)$ & $0.4,0.3)$ & $0.7,0.1)$ \\
\hline
\end{tabular}

The corresponding matrix of ranks is

\begin{tabular}{|l|l|l|l|l|}
\hline \multirow{2}{*}{ Persons } & \multicolumn{5}{|c|}{ Jobs } \\
\cline { 2 - 5 } & \multicolumn{1}{|c|}{1} & \multicolumn{1}{|c|}{2} & \multicolumn{1}{c|}{3} & \multicolumn{1}{|c|}{4} \\
\hline $\mathrm{A}$ & 0 & 0.591 & {$[0]$} & 0.504 \\
\hline $\mathrm{B}$ & 2.415 & {$[0]$} & 0 & 1.237 \\
\hline $\mathrm{C}$ & {$[0]$} & 0.826 & 0.626 & 0 \\
\hline $\mathrm{D}$ & 1.926 & 0.235 & 0.411 & {$[0]$} \\
\hline
\end{tabular}

Here the least number of lines through columns and rows which will cover all zeros of the matrix is 4 and hence the optimal assignment can be made. The optimal assignment is

$$
A \rightarrow 3, B \rightarrow 2, C \rightarrow 1, D \rightarrow 4
$$

The corresponding optimal minimum fuzzy cost is given by

$$
\tilde{c}_{13}+\tilde{c}_{22}+\tilde{c}_{31}+\tilde{c}_{44}=((15,23,27,37),(10,19,32,43) ; 0.4,0.3) .
$$

\section{Conclusion}

In this paper, a new algorithm has been developed for solving assignment problems with costs as generalized trapezoidal intuitionistic fuzzy numbers by using the given ranking method. There are several papers in the literature for solving assignment problems with intuitionistic fuzzy costs, but no one has used generalized intuitionistic fuzzy costs. The algorithm is easy to understand and can be used for all types of assignment problems with costs as fuzzy as well as intuitionistic fuzzy numbers. 


\section{References}

[1] Atanassov, K., Intuitionistic fuzzy sets, Fuzzy Sets and Systems, 20 (1986), $87-96$.

[2] Atanassov, K., More on intuitionistic fuzzy sets, Fuzzy Sets and Systems, 33 (1989), 37-46.

[3] Chen, M.S., On a fuzzy assignment problem, Tamkang Journal, 22 (1985), 407-411.

[4] Chen Liang-Hsuan, Lu Hai-Wen, An extended assignment problem considering multiple outputs, Applied Mathematical Modeling, 31 (2007), 22392248 .

[5] Lin Chi-Jen, Wen Ue-Pyng, A labeling algorithm for the fuzzy assignment problem, Fuzzy Sets and Systems, 142 (2004), 373-391.

[6] Liu, L. and Gao, X., Fuzzy weighted equilibrium multi-job assignment problem and genetic algorithm, Applied Mathematical Modeling, 33 (2009), 3926-3935.

[7] Mahapatra, G.S. and Mahapatra, B.S., Intuitionistic fuzzy fault tree analysis using intuitionistic fuzzy numbers, International Mathematical Forum, 5(21) (2010), 1015-1024.

[8] Majumdar, J. and Bhunia, A.K., Elistic genetic algorithm for assignment problem with imprecise goal, European Journal of Operations Research 177 (2007), 684-692.

[9] Nagoor Gani, A. and Mohamed, V.N., A method of ranking generalized trapezoidal intuitionistic fuzzy numbers, Int. J. Appl. Eng. Res. 10(10), 2015, 25465-25473.

[10] Sakawa, M., Nishizaki, I. and Uemura, Y., Interactive fuzzy programming for two level and linear fractional production and assignment problems; a case study, European Journal of Operations Research, 135 (2001), 142-157.

[11] Nirmala, G. and Anju, R., Cost minimization assignment problem using fuzzy quantifier, International Journal of Computer Science and Information Technologies, 5(6), 2014, 7948-7950.

[12] Pandian, P. and Kavitha, K., A new method for solving assignment problems, Annals of Pure and Applied Mathematics, 1(1) (2012), 69-83. 
[13] Sathi Mukherjee and Kajla Basu, Solving intuitionistic fuzzy assignment problem by using similarity measures and score functions, International Journal of Pure and Applied Science and Technologies, 2(1) (2011), 1-18.

[14] Shiny Jose and Sunny Kuriakose, A., Algorithm for solving assignment model in intuitionistic fuzzy context, International Journal of Fuzzy Mathematics and Systems, 3(5) (2013), 345-349.

[15] Stephen Dinagar, D. and Thiripurasundari, K., A novel method for solving fuzzy transportation problem involving intuitionistic trapezoidal fuzzy numbers, International Journal of Current Research, 6(6) (2014), 70387041.

[16] Thorani, Y.L.P. and Ravi Shankar, N., Fuzzy assignment problem with generalized fuzzy numbers, Applied Mathematical Sciences, 7(71) (2013), 3511-3537.

[17] Ye. X. and Xu, J., A fuzzy vehicle routing assignment model with connection network based on priority-based genetic algorithm, World Journal of Modeling and Simulation, 4 (2008), 257-268. 
